\title{
jinlong capsule inhibits migration and invasion in human glioblastoma cells via the modulation of mTOR/S6 signaling pathway
}

This article was published in the following Dove Press journal:

Drug Design, Development and Therapy

\author{
Jingren Shi' \\ Wenli Zhang ${ }^{2}$ \\ Lu $\mathrm{He}^{2}$ \\ Fanhong Kong ${ }^{2}$ \\ Meichen Pan' \\ Jingjing Guo' \\ Xinmin $X u^{\prime}$ \\ Jie Guo' \\ Huizhu Wang' \\ Yajie Wang' \\ 'Department of Clinical Laboratory, \\ Beijing Ditan Hospital, Capital Medical \\ University, Beijing I000I5, People's \\ Republic of China; ${ }^{2}$ Department of \\ Clinical Laboratory, Beijing Tiantan \\ Hospital, Capital Medical University, \\ Beijing 100050, People's Republic of \\ China
}

\begin{abstract}
Aim: To investigate the anticancer effects of Jinlong capsule (JLC) against human glioblastoma cells and the possible underlying mechanism.

Methods: Cell Counting Kit-8 and colony formation assay were adopted for the analysis of cell viability. Cell invasion and migration were evaluated by transwell and wound healing assays. Then, the expression level of mammalian target of rapamycin (mTOR), phosphorylated mTOR (pmTOR), S6 and phosphorylated S6 (p-S6) were determined by western blotting.

Results: The results showed that JLC significantly inhibited human glioblastoma cell proliferation, invasion and migration in a dose-dependent manner. The expressions of p-mTOR and p-S6 were dramatically suppressed by JLC. Furtherly, inhibition of mTOR reduced the cell migration and invasion, while the mTOR agonist (MHY1485) could partially reverse the anti-migration and anti-invasion activity of JLC.

Conclusion: The above results suggested that JLC would be a potential candidate for the treatment of glioblastoma.
\end{abstract}

Keywords: Jinlong capsule, invasion, migration, glioblastoma, mTOR, S6

\section{Introduction}

Glioblastoma is the most lethal type of brain tumor, accounting for $46.6 \%$ of primary malignant brain tumors. ${ }^{1}$ The standard therapeutic regimen remained maximal neurosurgical resection, radiotherapy and/or chemotherapy with temozolomide. ${ }^{2}$ In recent years, even though a variety of novel approaches to the treatment of glioblastoma have emerged, such as immunotherapy, ${ }^{3}$ gene therapy, ${ }^{4}$ transdifferentiation induced neural stem cells, ${ }^{5}$ stereotactic laser interstitial thermal therapy, ${ }^{6}$ and so on, the mean survival of glioblastoma patients is still 14.6 months, ${ }^{7}$ while the 5-year survival rate remains only $5.5 \%{ }^{1}$

The aggressive properties of glioblastoma, including uncontrolled proliferation, invasive growth and rapid migration, result in the obstacle to the treatment of glioblastoma. ${ }^{8}$ Therefore, it is essential to explore effective medicines and treatment strategies to prevent the proliferation, invasion and migration of glioblastoma. Recently, traditional Chinese medicine (TCM) has gained wide attention due to their therapeutic effects on glioblastoma and less side effects. More and more kinds of TCMs have been confirmed to have antitumor effects against glioblastoma. ${ }^{9-12}$

Jinlong capsule (JLC), consist of Gekko, Bungarus and Agkistrodon, is a TCM preparation which has been used for years. ${ }^{13}$ Its effective components were extracted by cryogenic freezing, biochemical separation, reverse osmosis concentration and
Department of Clinical Laboratory, Beijing Ditan Hospital, Capital Medical University, 8 Jingshun Dongjie, Beijing 100015, People's Republic of China Tel +86 1084322429

Email wangyajie@ccmu.edu.cn 
other technologies from the raw animal materials. ${ }^{14}$ One study has identified 17 kinds of amino acids by UPLC and established the fingerprint of amino acid composition of JLC. $^{15}$ Gekko sulfated polysaccharide-protein complex (GSPP), extracted from Gekko, have been proven to be a good antitumor preparation. ${ }^{16,17}$ Clinical researches find that JLC has anticancer effects in a way on a range of malignant tumors, and can improve the patient's immune function and survival quality. ${ }^{18-20}$ Preclinical study indicates that JLC can inhibit proliferation and induces apoptosis in a variety of cancer cells. ${ }^{13,14}$ But up to now, there are very few studies on the antitumor effect of JLC against glioblastoma. To the best of our knowledge, so far, there has only been one in vivo study on the anti-glioma effect of JLC, but the anticancer mechanism is not clear. ${ }^{21}$ In this study, we aimed to determine whether JLC inhibits the invasion and migration of human glioblastoma cells in vitro and explore the underlying mechanisms.

\section{Materials and methods}

\section{Drugs and reagents}

JLC was supplied by Beijing Jiansheng Pharmaceutical Co., Ltd. (No.170517, Beijing, China). JLC was stored at $-20^{\circ} \mathrm{C}$ and dissolved in DMEM at a concentration of $100 \mathrm{mg} / \mathrm{mL}$ as a stock solution, then diluted with DMEM before each experiment.

Antibodies to mammalian target of rapamycin (mTOR) (\#2983), S6 (\#2217) and p-S6 (\#4858) were purchased from Cell Signaling Technology (Boston, MA, USA). Antibody to p-mTOR (ab109268) was purchased from Abcam (Cambridge, MA, USA). Cell Counting Kit-8 (CCK-8) was obtained from Dojindo Molecular Technologies (Japan). Rapamycin and MHY1485 were purchased from MedChemExpress (New Jersey, USA).

\section{Cell culture}

Human glioblastoma cell lines A172 and U251 were purchased from the Cell Resource Center of Peking Union Medical College (Beijing, China). A172 and U251 cells were cultured in DMEM (Grand Island, NE, USA) supplemented with $10 \%$ FBS (Grand Island, NE, USA) at $37^{\circ}$ $\mathrm{C}$ with $5 \% \mathrm{CO}_{2}$ atmosphere.

\section{CCK-8 assay}

The cell viability was measured with CCK- 8 assays which is based on WST-8 (2-(2-methoxy-4-nitrophenyl)-3-(4-nitrophenyl)-5-(2,4-disulfonyl benzene)-2H- tetrazole monosodium salt). WST- 8 is a compound similar to MTT, which can be reduced by some dehydrogenases in mitochondria to form orange formazan in the presence of electron-coupled reagents. The more and faster the cell proliferation, the darker the color. For the same cells, there is a linear relationship between color and number of cells. In brief, cells were seeded into 96-well culture plates at a density of $5 \times 10^{3}$ cells per well with specified concentrations of JLC $(0,0.5,1$, 2, 4, 8 and $10 \mathrm{mg} / \mathrm{mL}$ ) and incubated for $24 \mathrm{hr}$. Then, $10 \mu 1$ of CCK- 8 was added to each well and cells were incubated at $37^{\circ} \mathrm{C}$ for $1 \mathrm{hr}$. The absorbance at $450 \mathrm{~nm}$ was determined using the microplate reader (BioRad, Hercules, CA, USA).

\section{Crystal violet assay}

A172 and U251 cells were seeded into 12-well culture plates at a density of $2 \times 10^{5}$ and $3 \times 10^{5}$ cells per well, respectively. After changed with specified concentrations of $\operatorname{JLC}(0,1,4,8$ and $10 \mathrm{mg} / \mathrm{mL})$, cells were incubated for $24 \mathrm{hr}$. The cells were fixed with $4 \%$ pre-cooling paraformaldehyde for $15 \mathrm{~min}$, stained with $0.1 \%$ crystal violet and photographed.

\section{Colony formation assay}

A172 and U251 cells were seeded into six-well culture plates at a density of 500 cells per well. Cells were changed with medium containing $0 \mathrm{mg} / \mathrm{mL}$ or $8 \mathrm{mg} / \mathrm{mL}$ JLC every 3 days and incubated for 12 days. After $4 \%$ precooling paraformaldehyde fixing for $15 \mathrm{~min}$, the colonies were stained with $0.1 \%$ crystal violet for photographing.

\section{Scratch wound healing assay}

A172 and U251 cells $\left(5 \times 10^{5}\right.$ cells in $2 \mathrm{ml}$ cell culture medium) were seeded into six-well plates until the cellular confluence reached approximately $80 \%$. Three separate scratching wounds were created with a sterile $200 \mu \mathrm{l}$ pipette tip. After rinsed with PBS (Grand Island, NE, USA) for three times, the medium was replaced with serum-free DMEM with or without JLC at different concentrations for $24 \mathrm{hr}$. Then, the wounds at marked lines were photographed and counted using Image $\mathrm{J}$ software (National Institutes of Health, Bethesda, MD, USA).

\section{Cell migration and invasion assay}

The effects of JLC on the migration and invasion were checked using transwell assay. Briefly, Cells at a density of $5 \times 10^{4}$ cells per well were seeded in the upper chamber of 
the transwell migration chambers $(8-\mu \mathrm{m}$ pore size; Costar, Cambridge, MA, USA) and incubated with $\operatorname{JLC}(0,1$ and $4 \mathrm{mg} / \mathrm{mL}$ ). The lower chamber was added with DMEM containing $20 \%$ FBS. After $24 \mathrm{hr}$, cells were fixed by $4 \%$ pre-cooling paraformaldehyde, stained with $0.1 \%$ crystal violet in methanol, and photographed in three independent fields for each well. Cell invasion assay was similarly operated with the migration assay except that coated Matrigel (BD Biosciences, San Jose, CA, USA) on the filter membrane. Migrating/invading cells were photographed and counted using an optical microscope (Carl Zeiss Meditec AG, Jena, Germany).

\section{Western blotting}

Cells were treated with JLC for $24 \mathrm{hr}$. The total protein was extracted with RIPA lysis buffer and phosphatase inhibitors (Applygen, Beijing, China). Equal quantity of protein was separated by SDS-PAGE gel and transferred onto polyvinylidene difluoride membranes (Millipore, Billerica, MA, USA). The membranes were blocked with $5 \% \mathrm{BSA}$ at room temperature for $1 \mathrm{hr}$, incubated with primary antibodies $(1: 1,000)$ at $4^{\circ} \mathrm{C}$ overnight and incubated with horseradish peroxidase (HRP)-conjugated secondary antibodies $(1: 2,000)$ at room temperature for $1 \mathrm{hr}$. Protein bands were detected by chemiluminescence HRP substrate (Millipore) and analyzed by Image $\mathbf{J}$ software (National Institutes of Health).

\section{Statistical analysis}

All data are described as the mean $\pm \mathrm{SD}$. All experiments were done in triplicate or more. ANOVA was performed to determine differences between multiple groups. A $P$-value $<0.05$ was considered statistically significant. Statistical analyses were performed with SPSS 17.0 statistical software (SPSS Inc., Chicago, IL, USA).

\section{Results}

\section{JLC inhibited the cell viability}

The inhibitory effects of JLC on the viability of glioblastoma cells were assessed by CCK- 8 assays and colony formation assay. A172 and U251 cells were treated with JLC for $24 \mathrm{hr}$ under a range of concentrations $(0,0.5,1,2$, 4, 8 and $10 \mathrm{mg} / \mathrm{mL}$ ). As shown in Figure 1A, cell viability was inhibited in a concentration-dependent manner when drug concentration was greater than or equal to $2 \mathrm{mg} / \mathrm{mL}$. After treated with $0,1,4,8$ and $10 \mathrm{mg} / \mathrm{mL}$ JLC, cells were visualized by light microscopy and stained with crystal violet to observe the changes in morphology. As the concentration of the drug increased, the cells exhibited cell vacuoles, shrinkage and turned round, especially in A172 cells (Figure 1B and C). Colony formation assay showed cell colonies were reduced after drug treatment (Figure 1D). The above results indicated that JLC could suppress glioma cells proliferation and cell viability. To avoid the inhibitory effect of JLC on cell invasion and migration caused by the decreased cell viability, the cell viability $>80 \%$, that is, concentrations of $1 \mathrm{mg} / \mathrm{mL}$ and $4 \mathrm{mg} / \mathrm{mL}$ were selected in all subsequent experiments unless otherwise indicated.

\section{JLC inhibited the mobility of AI72 and U25 I cells}

The effects of JLC on the mobility of A172 and U251 cells were checked by wound healing assays. A172 and U251 cells were treated with 0,1 and $4 \mathrm{mg} / \mathrm{mL}$ JLC for $24 \mathrm{hr}$. After creating scratching wounds with a $200 \mu$ pipette tip, the cell-free areas were detected. The results indicated that JLC inhibited the cell mobility of A172 and U251 cells substantially in a concentration-dependent manner compared with control group (Figure 2).

\section{JLC inhibited invasion and migration of AI72 and U25I cells}

Next, the effects of JLC on the invasion and migration of A172 and U251 cells were assessed with transwell assays with or without matrigel. As illustrated in Figure 3, the number of cells with invasion and migration reduced remarkably with the concentration of JLC increased, suggesting JLC can inhibit the invasion and migration of A172 and U251 cells.

\section{JLC suppresses $\mathrm{mTOR} / \mathrm{S} 6$ signaling in AI72 and U25I cells}

Our results showed that JLC inhibits migration and invasion activity in A172 and U251 cells dramatically. It has been revealed that mTOR plays an important role in regulation of tumor cell motility, invasion and cancer metastasis, including glioma cells. ${ }^{22,23}$ Therefore, we investigated whether the effects of JLC on cell migration and invasion could be ascribed to the possible involvement of mTOR signaling pathway. The results showed that JLC reduced phosphorylation of mTOR and $\mathrm{S} 6$ in a concentration-dependent manner, while no significant differences were detected in the expression of the total protein levels of these molecules (Figure 4). 

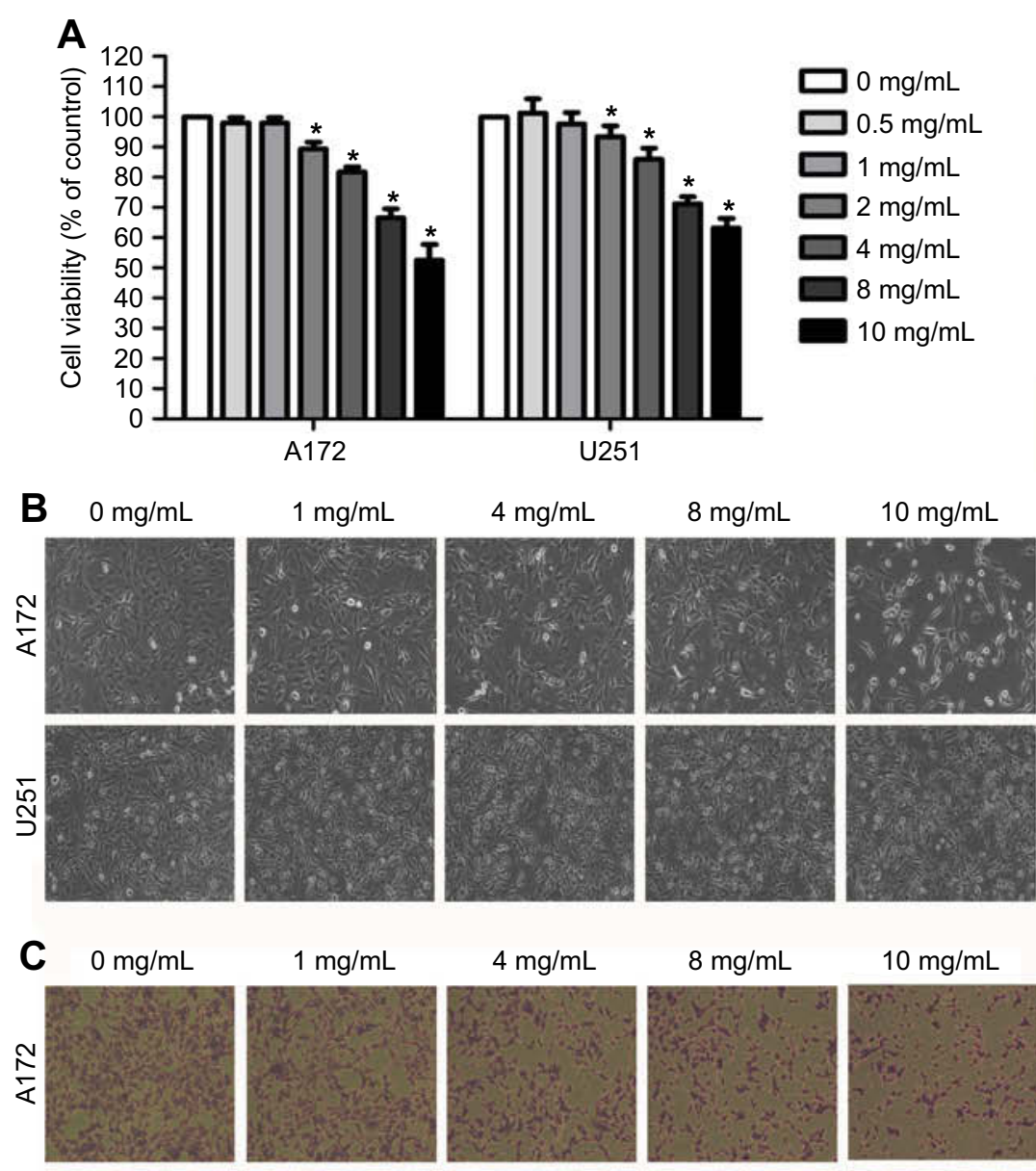

$1 \mathrm{mg} / \mathrm{mL}$
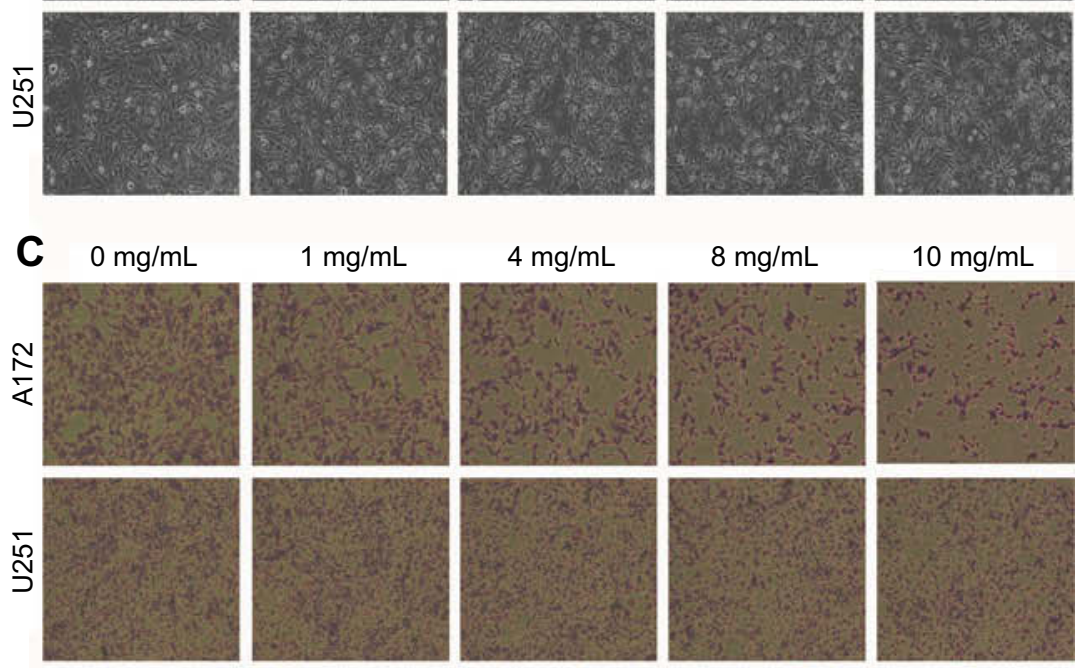

$10 \mathrm{mg} / \mathrm{mL}$
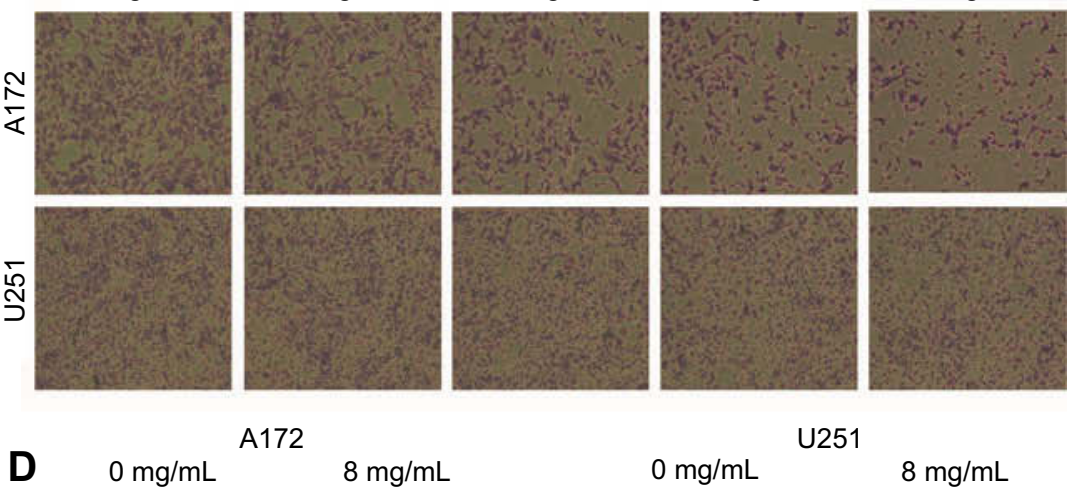

A172

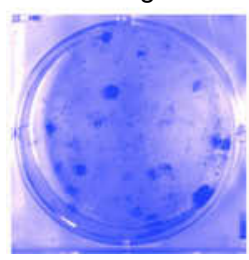

$8 \mathrm{mg} / \mathrm{mL}$
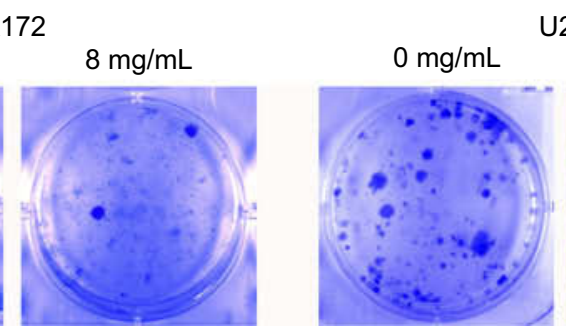

U251

$8 \mathrm{mg} / \mathrm{mL}$

Figure I Effect of JLC on the viability of glioblastoma cells. The cells were treated with different concentrations of JLC and examined using a CCK-8 assay (A), light microscope (B), crystal violet staining (C), and colony formation assay (D). Data were represented as the mean \pm SEM of three independent experiments. $* P<0.05$, vs untreated group. Magnification, $100 \times$.

Abbreviations: JLC, Jinlong capsule; CCK, Cell Counting Kit-8.

\section{JLC inhibits invasion and migration} activity by blocking $\mathrm{mTOR} / \mathrm{S} 6$ pathways in AI72 and U25I cells

To further confirm whether the inhibition of invasion and migration activity by JLC mainly depend on its suppression of mTOR/S6 pathways, A172 and U251 cells were treated with JLC (4 mg/mL), mTOR inhibitor rapamycin (Rapa, $10 \mu \mathrm{M}$ ) alone, or combination of JLC (4 mg/mL) and MHY1485 (a mTOR agonist, 10 $\mu \mathrm{M})$. The results indicated that inhibitor of mTOR could inhibit the expression of p-mTOR and p-S6, simultaneously reduced the cell migration and invasion dramatically, while MHY1485 could improve the level 
A

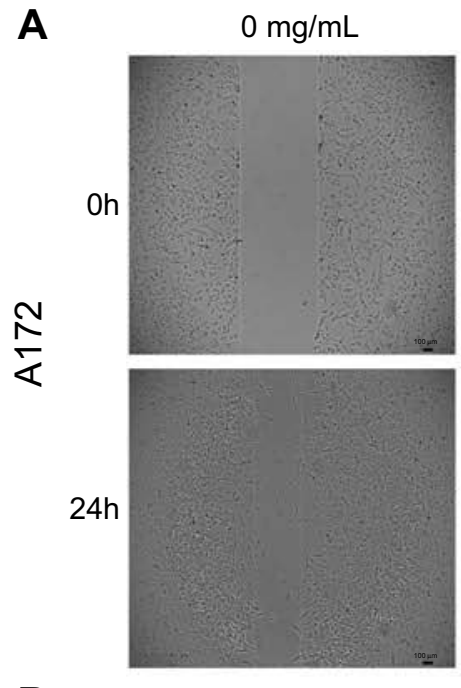

B

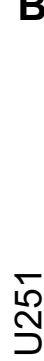

$1 \mathrm{mg} / \mathrm{mL}$
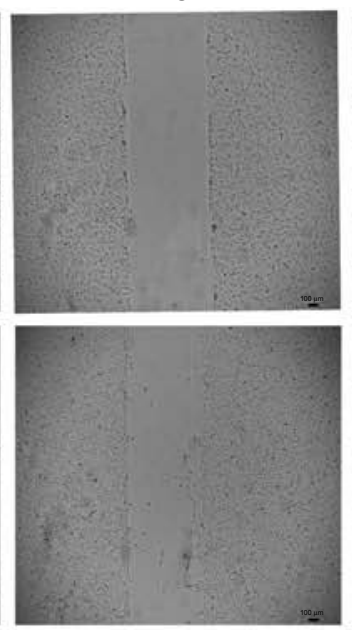

$1 \mathrm{mg} / \mathrm{mL}$
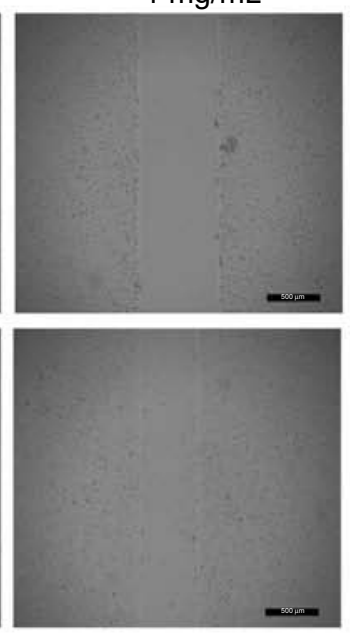

D
$4 \mathrm{mg} / \mathrm{mL}$

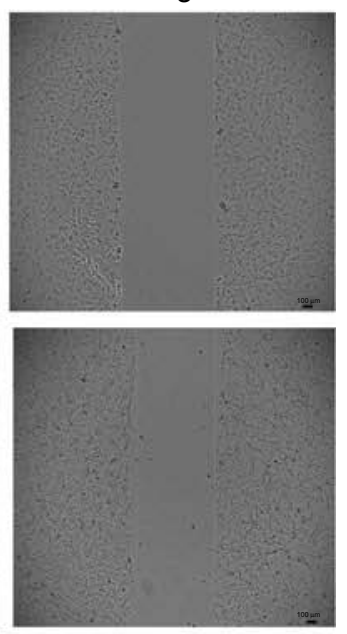

$4 \mathrm{mg} / \mathrm{mL}$
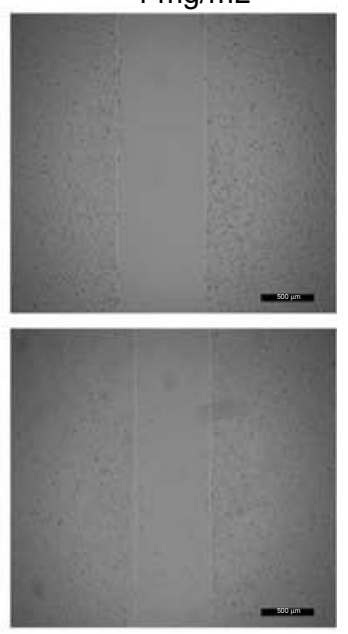

U251
C

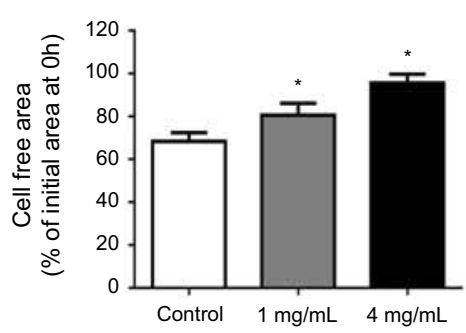

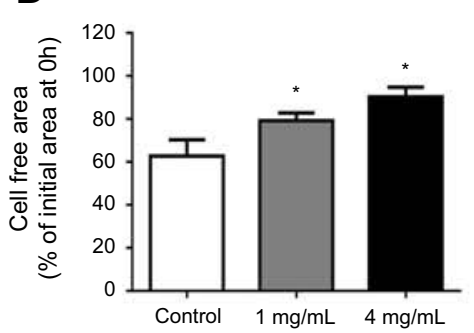

Figure 2 JLC inhibited the wound closure of AI72 and U25I cells. (A) Representative images of wound closures in AI72 cells. (B) Representative images of wound closures in U25I cells. (C) Statistical analysis of cell-free area for AI72 cells. (D) Statistical analysis of cell-free area for U25I cells. All experiments were performed in triplicates. $* P<0.05$ (compared with control group). Magnification, 40x.

Abbreviation: JLC, Jinlong capsule.

of p-mTOR and p-S6, and partially remedy the inhibitory effect of JLC on cell migration and invasion (Figure 5). On the basis of the above results, it can be suggested that the cell migration and invasion of A172 and U251 cells should be restrained by JLC, at least in part, through the mTOR/S6 pathway.

\section{Discussion}

Glioblastoma is the primary malignant tumor in the central nervous system, ${ }^{1}$ and surgical resection is still the main treatment at present. $^{2}$ Due to the characteristics of invasive growth and strong migration, the recurrence rate of glioblastoma is very high. ${ }^{24,25}$ Cell migration and invasion 


\section{A}

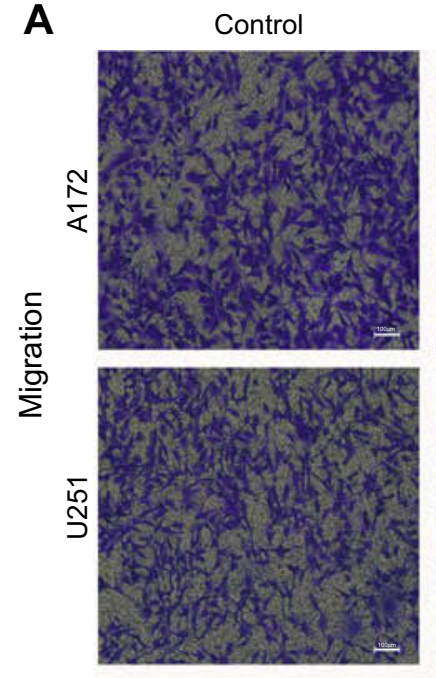

B

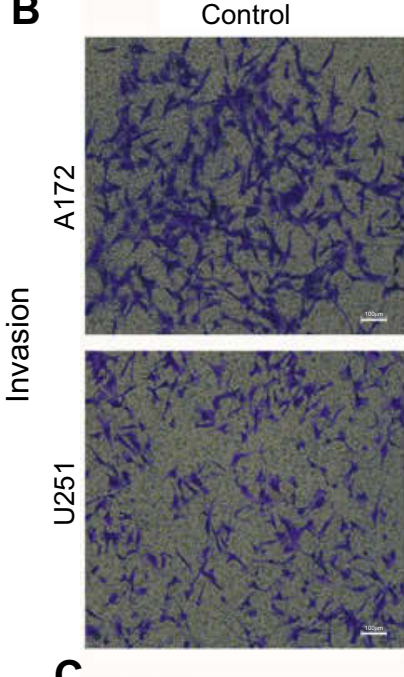

C

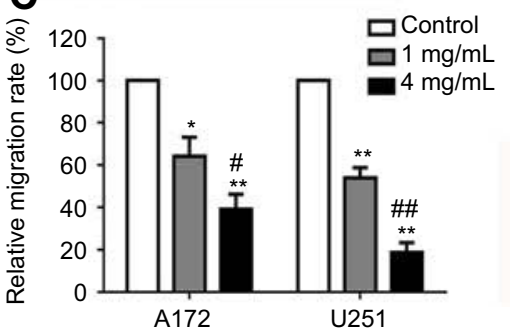

$1 \mathrm{mg} / \mathrm{mL}$
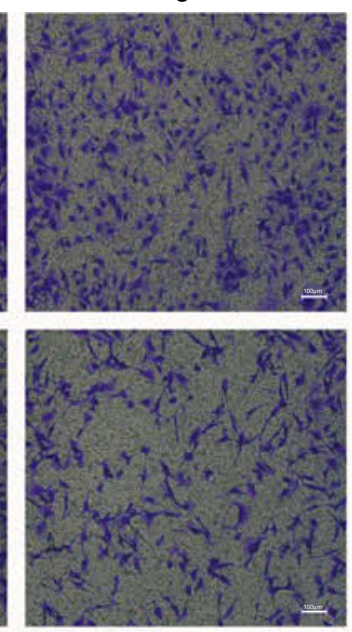

$1 \mathrm{mg} / \mathrm{mL}$
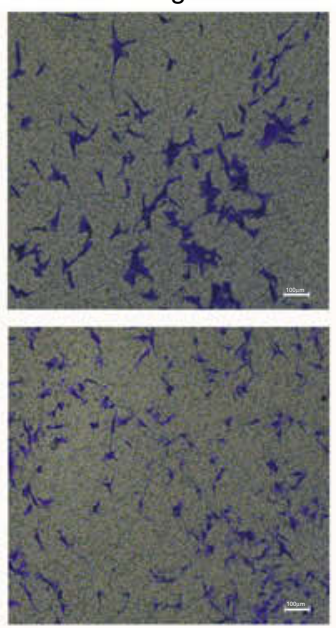

D

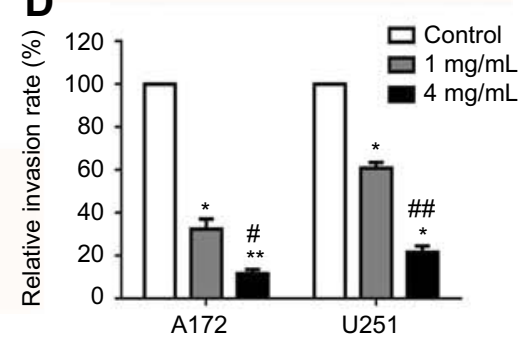

Figure 3 JLC inhibited the invasion and migration of AI72 and U25I cells. (A) Representative images of migration in AI72 and U25I cells. (B) Representative images of invasion in AI72 and $\mathrm{U} 25 \mathrm{I}$ cells. (C) Statistical analysis of relative migration rate. (D) Statistical analysis of relative invasion rate. All experiments were performed in triplicate. ${ }^{* P}<0.05,{ }^{*} * P<0.01$ (compared with control groups), ${ }^{\#} P<0.05,{ }^{\# \#} P<0.01$ (compared with I mg/mL groups). Magnification, $40 \times$.

Abbreviation: JLC, Jinlong capsule.

play critical roles in the metastasis of cancer cells, which can be attributed to the activation of relevant target signaling pathways and contribute to tumor recurrence. Therefore, it is pivotal to inhibit cancer cell migration/ invasion and suppress the activation of the target signaling pathway. ${ }^{26}$ Recently, accumulating researches have verified that a wide variety of TCMs reveal inhibitory effects on cancer cells including glioblastoma cells. For example, hedyotis diffusa willd extract inhibits the growth of human glioblastoma cells in a dose- and time-dependent manner. ${ }^{27}$ Shikonin can attenuate the proliferation, migration, and invasion capability of human glioblastoma cells. ${ }^{10}$ Toosendanin inhibits GBM cell proliferation and induces apoptosis in vitro and in vivo. ${ }^{12}$ Calycosin inhibits 

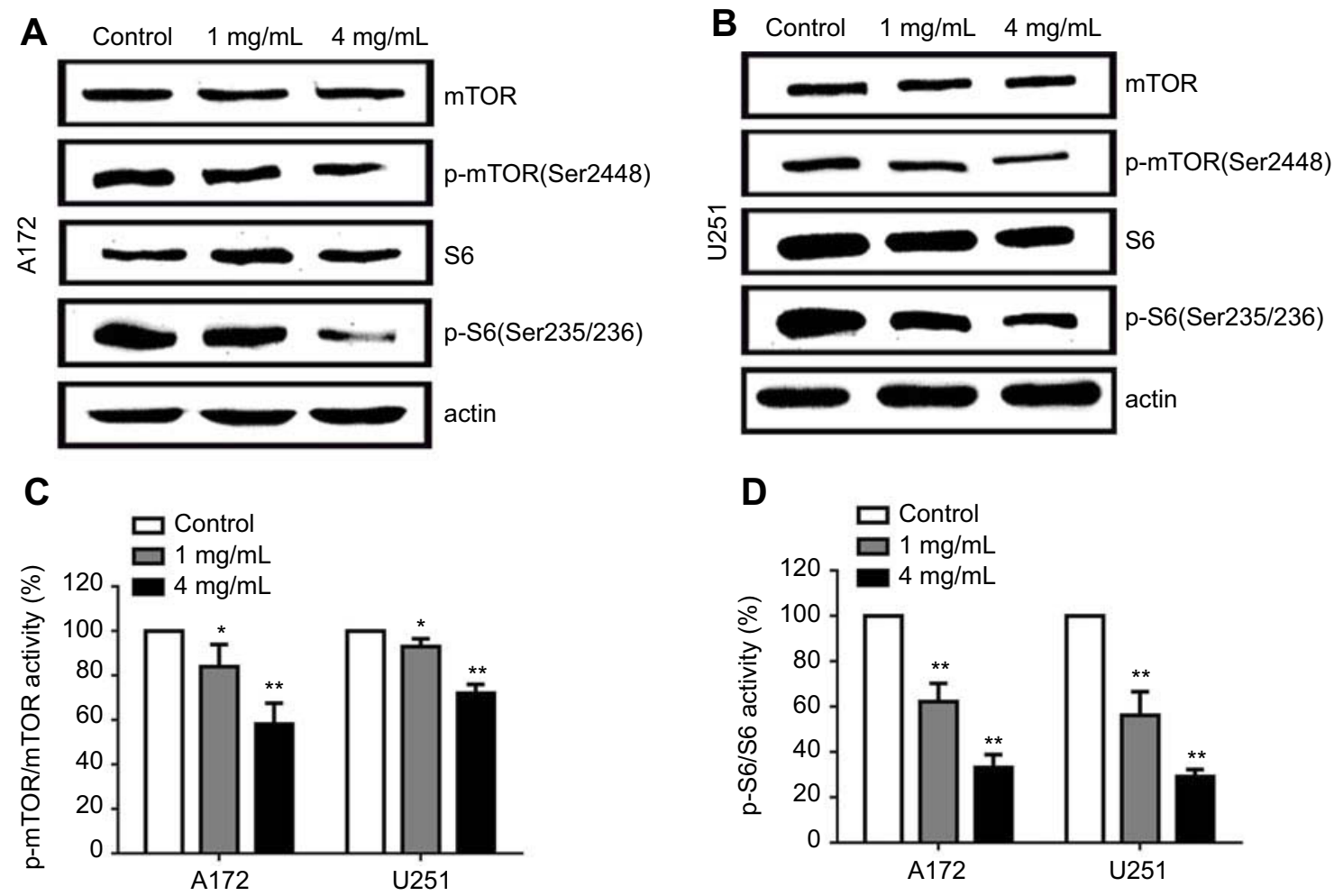

Figure 4 Effects of JLC on mTOR/S6 pathway in AI72 and U25I cells. (A) Representative blot of AI72 cell lysates. (B) Representative blot of U25I cell lysates. (C) The band intensities of $\mathrm{p}-\mathrm{mTOR}$ relative to total mTOR. (D) The band intensities of $\mathrm{p}-\mathrm{S} 6$ relative to total S6. All experiments were performed in triplicates. Significant difference from control group, $* P<0.05$ and $* * P<0.01$.

Abbreviations: JLC, Jinlong capsule; mTOR, mammalian targetof rapamycin.

migration and invasion in U87 and U251 cells. ${ }^{28}$ These studies indicate that TCMs have potential value in the treatment of glioblastoma.

JLC and one of its active ingredients-GSPP have significant effects of anti-tumor. ${ }^{16-20}$ Recently, one in vivo study showed that JLC could inhibit the growth of orthotopic glioblastoma in the nude mice without evident side effect. ${ }^{21}$ It could be the indirect evidence that JLC could go through the blood-brain barrier which is the major challenge of drugs to cure glioblastoma, and illustrated that JLC has potential clinical prospect in the treatment of glioblastoma. The mechanism of antitumor activities of JLC has not been elucidated thoroughly now, however. In this study, we investigated the anticancer effects of JLC against human glioblastoma cells in vitro and explored the possible mechanism, in order to discover a new strategy for glioblastoma.

Firstly, we examined the anti-proliferative ability of JLC against human glioblastoma A172 and U251 cell lines with CCK-8 and colony formation assay. The results showed that JLC could inhibit the proliferation and cell viability of glioma cells, especially in A172 cells. This discrepancy may be due to the different sensitivities to the drug in specific glioblastoma cell type. To ensure the inhibitory effects of JLC on cell migration and invasion were not caused by the cytotoxicity of the compound, concentrations of $1 \mathrm{mg} / \mathrm{mL}$ and $4 \mathrm{mg} / \mathrm{mL}$ were used in subsequent experiments. Next, transwell assays and wound healing assays were carried out to investigate the effects of JLC on cell migration and invasion. Data showed that JLC inhibited the migration and invasion of A172 and U251 cell lines in a dose-dependent manner (Figures 2 and 3). Our results are in coherence with nearest report. ${ }^{21}$

Another focus of attention for us was the mechanisms involved in this process. It has been reported that the mTOR not only can impact cell growth, survival and differentiation, ${ }^{29}$ but also plays a vital role in regulating tumor cell motility, invasion and cancer metastasis. ${ }^{30-32}$ mTOR contains two distinct complexes, named mTOR complex 1 (mTORC1) and 2 (mTORC2), in which the activation of mTORC1 leads to the phosphorylation of p70S6 kinase (S6K) and eukaryotic initiation factor 4E (eIF4E) binding protein 1 (4E-BP1). ${ }^{33} \mathrm{~S} 6$ is a downstream target of S6K1, which is activated by phosphorylation. A growing body of evidence pointed that mTOR signal was often activated in glioma, making it a therapeutic 


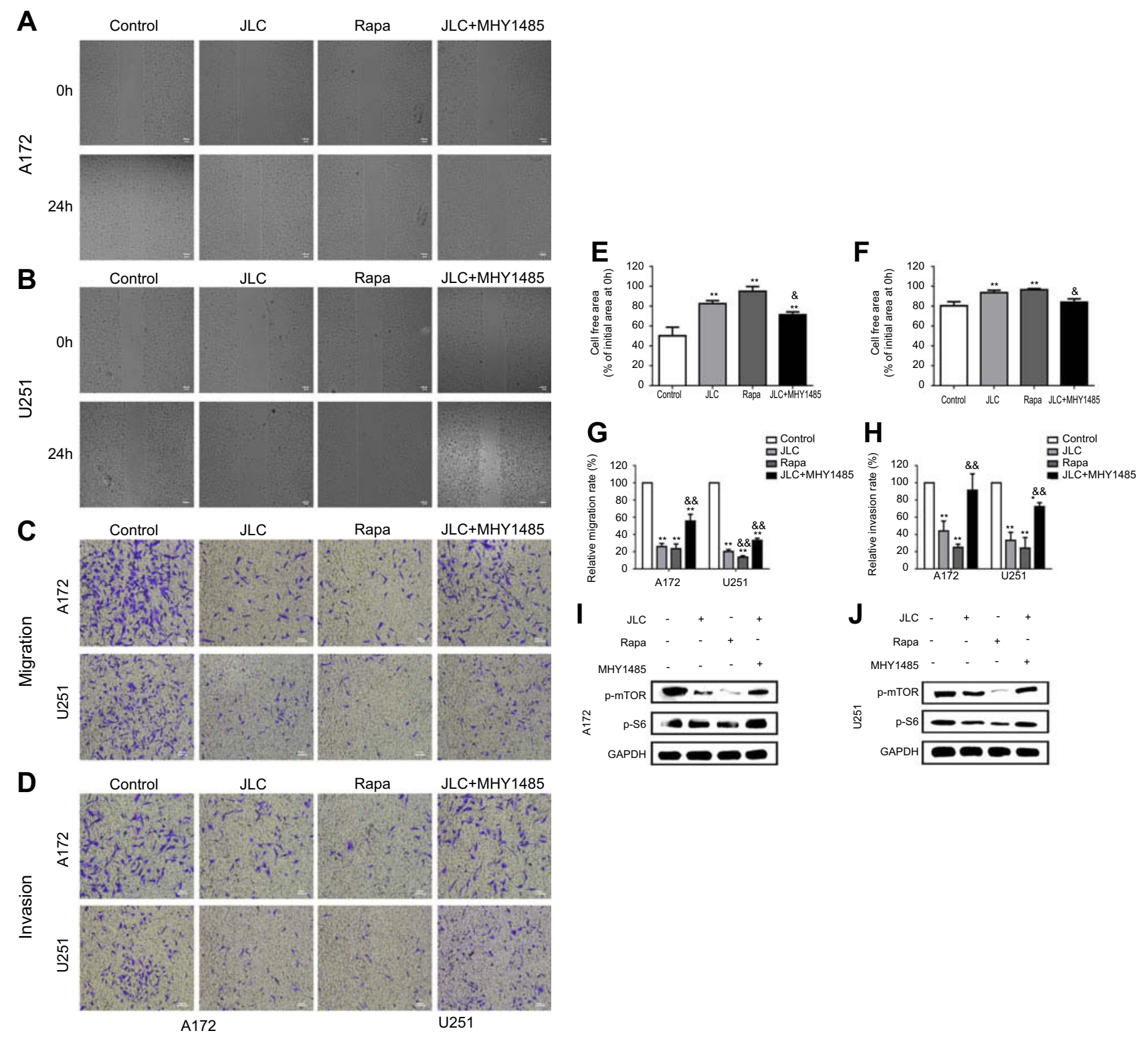

Figure 5 Effects of rapamycin and MHYI485 on the invasion and migration of AI72 and U25I cells. (A) Representative images of wound closures in AI72 cells. (B) Representative images of wound closures in U25I cells. (C) Representative images of migration in AI72 and U25I cells. (D) Representative images of invasion in AI72 and U25I cells. (E) Statistical analysis of cell-free area for AI72 cells. (F) Statistical analysis of cell-free area for U25I cells. (G) Statistical analysis of relative migration rate. (H) Statistical analysis of relative invasion rate. (I) Representative blot of AI72 and U25I cell lysates. All experiments were performed in triplicates. Significant difference from control group, $* P<0.05$ and $* * P<0.01$. Significant difference from JLC group, ${ }^{\circledR} P<0.05$ and ${ }^{\& \&} P<0.01$. Magnification, $40 \times$.

Abbreviation: JLC, Jinlong capsule.

target. $^{34,35}$ The inhibitors of mTOR can suppress the proliferation, invasion and migration of glioma cells. ${ }^{22,36}$ Besides, clinical studies found that there was an inverse relationship between the expression of phospho-S6 and survival of glioma patients. ${ }^{34}$ The above research results indicate that $\mathrm{mTOR}$ and S6 play a critical role in the occurrence and metastasis of glioma. To verify whether JLC exerts the effect of antiinvasion and anti-migration by regulating the mTOR/S6 pathway, the expression levels of mTOR and S6 proteins were detected by Western blotting assays. The results revealed that JLC only inhibited the expression of phosphorylated mTOR and phosphorylated S6 in a concentration-dependent manner, while did not influence the expression level of total protein in the cells (Figure 4). To further support these findings, cell invasion and migration were examined upon the treatment of mTOR inhibitor or JLC in combination with mTOR agonist. Our results demonstrated that inhibition of mTOR reduced the cell migration and invasion, while mTOR agonist could 
partially remedy the inhibitory effect of JLC on cell migration and invasion (Figure 5).

The data taken together in this study indicated that JLC regulates, at least in part, the $\mathrm{mTOR} / \mathrm{S} 6$ pathway to exert the inhibitive effects of JLC on migration and invasion in glioblastoma. But, there are a number of limitations in the current study. First, we only studied in cellular level without the support of clinical samples and animal experiments, the anti-glioma effect of JLC needs further verification. Second, this study lacks the research on the effect of JLC on normal human astrocytes. Furthermore, mTOR is involved in the transmission of multiple signal pathways, including PI3K/ AKT/mTOR pathway, AKT/TSC1-TSC2/Rheb/mTOR pathway and LKB1-AMPK-TSC-mTOR pathway, etc., all of which can affect its downstream target S6K1 and 4E-BP1, so as to achieve the regulation of cellular functions. ${ }^{37}$ However, the exact upstream pathway through which JLC regulates mTOR remains unclear. Besides, JLC as a Chinese traditional compound medicine, it is necessary to further elucidate its active components in anti-glioma. Therefore, further researches with the help of high-throughput and bioinformatics are needed to explore its exact active ingredient and mechanism(s).

\section{Conclusion}

The data taken together in this study indicate that JLC regulates, at least in part, the $\mathrm{mTOR} / \mathrm{S} 6$ pathway to exert the inhibitive effects of JLC on migration and invasion in glioblastoma in vitro. Although some limitations lay in the research, and further work is still needed to be investigated. Anyway, one thing it means is that JLC has potential as a candidate for the treatment of glioblastoma in the future. This study also provides further evidence for the treatment of glioma with TCM.

\section{Data availability}

The data of this study are available from the corresponding author on reasonable request.

\section{Acknowledgment}

This work was supported by the National Natural Science Foundation of China (No. 81572474) and the Science and Technology Development Fund Project of Traditional Chinese Medicine of Beijing (No. JJ2015-14).

\section{Disclosure}

The authors report no conflicts of interest in this work.

\section{References}

1. Ostrom QT, Gittleman H, Xu J, et al. CBTRUS statistical report: primary brain and other central nervous system tumors diagnosed in the United States in 2009-2013. Neuro Oncol. 2016;18(Suppl_5):v1v75. doi:10.1093/neuonc/now207

2. Stupp R, Hegi ME, Mason WP, et al.; European Organisation for Research and Treatment of Cancer Brain Tumour and Radiation Oncology Groups, National Cancer Institute of Canada Clinical Trials Group. Effects of radiotherapy with concomitant and adjuvant temozolomide versus radiotherapy alone on survival in glioblastoma in a randomised phase III study: 5-year analysis of the EORTC-NCIC trial. Lancet Oncol. 2009;10 (5):459-466. doi:10.1016/S1470-2045(09)70234-7.

3. Bloch O. Immunotherapy for malignant gliomas. Cancer Treat Res. 2015;163:143-158. doi:10.1007/978-3-319-12048-5_9

4. Strebe JK, Lubin JA, Kuo J. "Tag Team" glioblastoma therapy: results from a phase 1 trial of toca 511 and 5-fluorocytosine for recurrent high-grade Glioma. Neurosurgery. 2016;79(6):18-20. doi:10.1227/01.neu.0000508605.38694.fd

5. Chakraborty S, Schneider J, Boockvar JA. Transdifferentiation-induced neural stem cells for the treatment of malignant gliomas. Neurosurgery. 2016;79(4):12-13. doi:10.1227/01.neu.0000499705.20596.5d

6. Lee I, Kalkanis S, Hadjipanayis CG. Stereotactic laser interstitial thermal therapy for recurrent high-grade gliomas. Neurosurgery. 2016;79:S24-S34. doi:10.1227/NEU.0000000000001443

7. Maugeri R, Schiera G, Di Liegro C, Fricano A, Iacopino D, Di Liegro I. Aquaporins and brain tumors. Int J Mol Sci. 2016;17 (7):1029. doi:10.3390/ijms17071029

8. Wang YB, Hu Y, Li Z, et al. Artemether combined with shRNA interference of vascular cell adhesion molecule-1 significantly inhibited the malignant biological behavior of human glioma cells. PLoS One. 2013;8(4):e60834. doi:10.1371/journal.pone.0060834

9. Cha JH, Choi YJ, Cha SH, Choi CH, Cho WH. Allicin inhibits cell growth and induces apoptosis in U87MG human glioblastoma cells through an ERK-dependent pathway. Oncol Rep. 2012;28(1):41-48. doi:10.3892/or.2012.1772

10. Zhang FY, Hu Y, Que ZY, et al. Shikonin inhibits the migration and invasion of human glioblastoma cells by targeting phosphorylated beta-catenin and phosphorylated PI3K/Akt: a potential mechanism for the anti-glioma efficacy of a traditional Chinese herbal medicine. Int J Mol Sci. 2015;16(10):23823-23848. doi:10.3390/ijms161023823

11. Liu F, Wang B, Wang J, et al. Oxymatrine inhibits proliferation and migration while inducing apoptosis in human glioblastoma cells. Biomed Res Int. 2016;2016:1-7. doi:10.1155/2016/2816056

12. Cao L, Qu D, Wang H, et al. Toosendanin exerts an anti-cancer effect in glioblastoma by inducing estrogen receptor beta- and p53-mediated apoptosis. Int J Mol Sci. 2016;17(11):1928. doi: $10.3390 / \mathrm{ijms} 17111928$

13. Li D, Ni T, Tao L, et al. Jinlong Capsule (JLC) inhibits proliferation and induces apoptosis in human gastric cancer cells in vivo and in vitro. Biomed Pharmacother. 2018;107:738-745. doi:10.1016/j. biopha.2018.08.049

14. Li Y, Hu J, Huang H, He Y. Effect of Jinlong capsule on proliferation and apoptosis of human pancreatic cancer cells BxPC-3. J Tradit Chin Med. 2013;33(2):205-210.

15. Li J, Xiao ST. Fingerprint establishment of amino acids composition of Jinlong Capsule. Chin J Inf Tradit Chin Med. 2014;21 (3):62-64. in Chinese.

16. Chen D, Yao WJ, Zhang XL, et al. Effects of Gekko sulfated polysaccharide-protein complex on human hepatoma SMMC-7721 cells: inhibition of proliferation and migration. $J$ Ethnopharmacol. 2010;127(3):702-708. doi:10.1016/j.jep.2009.12.003

17. Wu XZ, Chen D, Han XQ. Anti-migration effects of gekko sulfated glycopeptide on human hepatoma SMMC-7721 cells. Molecules. 2011;16(6):4958-4970. doi:10.3390/molecules 16064958 
18. Zhang HJ, Yang JJ, Wang WX, et al. Effects of Jinlong capsule on expressions of interleukin-2 and soluble interleukin-2 receptor in patients with primary liver cancer after transarterial chemoembolization therapy. Zhong Xi Yi Jie He Xue Bao. 2008;6(9):907-910. doi:10.3736/jcim20080906

19. Wu GL, Zhang L, Li TY, Chen J, Yu GY, Li JP. Short-term effect of combined therapy with Jinlong Capsule and transcatheter arterial chemoembolization on patients with primary hepatic carcinoma and its influence on serum osteopontin expression. Chin J Integr Med. 2010;16(2):109-113. doi:10.1007/s11655-010-0109-9

20. Lu Q, Luo JB, Feng YF, She Q, Shi ZF. Jinlong capsule combined with chemoradiotherapy for NSCLC: a meta-analysis. Zhongguo Zhong Yao Za Zhi. 2015;40(22):4491-4496.

21. Huang H, Cui XW, Yue GJ, Qu YY, Li JS. Inhibitory effect of Jinlong capsules on Gliomatosis cerebri and mechanisms. Pharm J Chin PLA. 2014;30(3):188-191. in Chinese.

22. Zhou H, Huang S. Role of mTOR signaling in tumor cell motility, invasion and metastasis. Curr Protein Pept Sci. 2011;12(1):30-42.

23. Chandrika G, Natesh K, Ranade D, Chugh A, Shastry P. Suppression of the invasive potential of glioblastoma cells by mTOR inhibitors involves modulation of $\mathrm{NF \kappa B}$ and PKC- $\alpha$ signaling. Sci Rep. 2016;6:22455. doi:10.1038/srep22455

24. De Bonis P, Fiorentino A, Anile C, et al. The impact of repeated surgery and adjuvant therapy on survival for patients with recurrent glioblastoma. Clin Neurol Neurosurg. 2013;115(7):883-886. doi:10.1016/j.clineuro.2012.08.030

25. Yong RL, Wu T, Mihatov N, et al. Residual tumor volume and patient survival following reoperation for recurrent glioblastoma. $J$ Neurosurg. 2014;121(4):802-809. doi:10.3171/2014.6.JNS132038

26. Fenteany G, Zhu S. Small-molecule inhibitors of actin dynamics and cell motility. Curr Top Med Chem. 2003;3:593-616. doi:10.2174/ 1568026033452348

27. Zhang Y, Xie RF, Xiao QG, Li R, Shen XL, Zhu XG. Hedyotis diffusa Willd extract inhibits the growth of human glioblastoma cells by inducing mitochondrial apoptosis via AKT/ERK pathways. J Ethnopharmacol. 2014;158(Pt A):404-411. doi:10.1016/j.jep.2014.10.017

28. Nie XH, Ou-Yang J, Xing Y, Li DY, Liu RE, Xu RX. Calycosin inhibits migration and invasion through modulation of transforming growth factor beta-mediated mesenchymal properties in U87 and U251 cells. Drug Des Devel Ther. 2016;10:767-779. doi:10.2147/ DDDT.S90457

29. Tian T, Nan KJ, Guo H, et al. PTEN inhibits the migration and invasion of HepG2 cells by coordinately decreasing MMP expression via the PI3K/Akt pathway. Oncol Rep. 2010;23 (6):1593-1600.

30. Busch S, Renaud SJ, Schleussner E, Graham CH, Markert UR. mTOR mediates human trophoblast invasion through regulation of matrix-remodeling enzymes and is associated with serine phosphorylation of STAT3. Exp Cell Res. 2009;315(10):1724-1733. doi:10.1016/j.yexcr.2009.01.026

31. Chen JS, Wang Q, Fu XH, et al. Involvement of PI3K/PTEN/AKT/ mTOR pathway in invasion and metastasis in hepatocellular carcinoma: association with MMP-9. Hepatol Res. 2009;39(2):177-186. doi:10.1111/j.1872-034X.2008.00449.x

32. Kuo HP, Lee DF, Chen CT, et al. ARD1 stabilization of TSC2 suppresses tumorigenesis through the mTOR signaling pathway. Sci Signal. 2010;3(108):ra9. doi:10.1126/scisignal.2000590

33. Riobo NA, Lu K, Ai X, Haines GM, Emerson CP. Phosphoinositide 3-kinase and Akt are essential for sonic hedgehog signaling. Proc Natl Acad Sci USA. 2006;103(12):4505-4510. doi:10.1073/ pnas.0504337103

34. McBride SM, Perez DA, Polley MY, et al. Activation of PI3K/mTOR pathway occurs in most adult low-grade gliomas and predicts patient survival. J Neurooncol. 2009;97(1):33-40. doi:10.1007/s11060-0090004-4

35. Gini B, Zanca C, Guo D, et al. The mTOR kinase inhibitors, CC214-1 and CC214-2, preferentially block the growth of EGFRvIII-activated glioblastomas. Clin Cancer Res. 2013;19 (20):5722-5732. doi:10.1158/1078-0432.CCR-13-0527

36. Chheda MG, Wen PY, Hochberg FH, et al. Vandetanib plus sirolimus in adults with recurrent glioblastoma: results of a phase I and dose expansion cohort study. J Neurooncol. 2015;121(3):627-634. doi:10.1007/s11060-014-1680-2

37. Laplante M, Sabatini DM. mTOR signaling in growth control and disease. Cell. 2012;149(2):274-293. doi:10.1016/j.cell.2012.0 3.017

\section{Publish your work in this journal}

Drug Design, Development and Therapy is an international, peerreviewed open-access journal that spans the spectrum of drug design and development through to clinical applications. Clinical outcomes, patient safety, and programs for the development and effective, safe, and sustained use of medicines are a feature of the journal, which has also been accepted for indexing on PubMed Central. The manuscrip management system is completely online and includes a very quick and fair peer-review system, which is all easy to use. Visit http://www. dovepress.com/testimonials.php to read real quotes from published authors. 